\title{
Implant-associated cracked teeth: case series
}

\author{
Eyal Rosen ${ }^{1 *}$, Tomer Goldberger ${ }^{1}$, Aviad Tamse $^{1}$, Carlos E. Nemcovsky², Salmon Breslauer ${ }^{3}$, Ilan Beitlitum $^{2}$ \\ and Igor Tsesis ${ }^{1}$
}

\begin{abstract}
Background: This study presents a series of 18 cases of cracked non-endodontically treated teeth that were diagnosed following the placement of adjacent implant-supported rehabilitation. These findings could suggest a possible cause/ related events.

Results: Most of the cases occurred in female patients (61\%), over 50 years old (78\%, with an average age of 59 years). Nine (50\%) of the cracked teeth were molars, 7 (39\%) were mandibular premolars, and 2 (11\%) were incisor teeth. Most of the cracked teeth (61\%) had an amalgam restoration, 50\% had deep periodontal pockets, and $78 \%$ had vital pulps. In $78 \%$ of patients, multiple implants were placed (with an average of 3 implants per patient). The time from implant loading to the diagnosis of a crack was longer than 1 year in $78 \%$ of cases.

Conclusions: The most common patient profile found in this series was a woman over 50 years old, having a cracked mandibular premolar tooth, which was diagnosed more than 1 year after reconstruction based on multiple adjacent implants. Additional clinical studies are warranted in order to shed light on this potential complication.
\end{abstract}

Keywords: Implant, Cracked teeth, Complications

\section{Background}

Implant-supported rehabilitation has become common practice for tooth replacement as a result of trauma, dental caries, periodontal disease, or for cases of a congenitally missing tooth (Mericske-Stern et al. 2001; Rosen et al. 2016). Implants anchorage to bone (osseointegration) (Depprich et al. 2008) is achieved by close contact between bone and implant (Novaes et al. 2010). Differing from natural teeth, implants lack the periodontal ligament (PDL) (Jackson 2003). The PDL possesses unique characteristics and enables mobility, stress dissemination, and tolerance to occlusal loads and proprioception, thus acting as a modulator of excessive occlusal loads. This stress modulation mechanism is absent in osseointegrated implants, which may potentially expose them to occlusal overload (Rosen et al. 2016; Jackson 2003).

Although the exact effects of occlusal overload on implants are not yet fully clear (Chambrone et al. 2010; Gotfredsen et al. 2001), it has been suggested that the occlusal

\footnotetext{
* Correspondence: Dr.eyalrosen@gmail.com

${ }^{1}$ Department of Endodontology, The Maurice and Gabriela Goldschleger

School of Dental Medicine, Tel Aviv University, Tel Aviv, Israel

Full list of author information is available at the end of the article
}

design of implant-supported rehabilitations should reduce these forces (Koyano and Esaki 2014). On the other hand, altering the occlusion of an implant-supported rehabilitation to prevent overload might increase the forces on the adjacent natural teeth (Rosen et al. 2016; Misch 1993; Engelman 1996).

Under excessive occlusal loads, teeth may be at risk of ensuing fractures (Kim et al. 2013; Lynch and McConnell 2002). Longitudinal teeth fractures include vertical root fractures (VRFs) that initiate from the root at any level (Rosen et al. 2016) and cracked teeth that are associated with complete or incomplete fractures initiating from the crown and extending subgingivally (Kang et al. 2016). While the exact occlusal relationship between implants and adjacent natural teeth is not fully elucidated (Lynch and McConnell 2002; Seo et al. 2012; Morgano and Brackett 1999; Goodacre and Spolnik 1994; Robbins 1990), a recent report (Rosen et al. 2016) suggested a possible association between the presence of an implant and the development of VRFs in adjacent endodontically treated teeth. However, the risk of implant-related cracked teeth is unknown. 
It may be hypothesized that the risk of cracks is higher in patients presenting with both implant-supported rehabilitations and adjacent natural teeth ("implant-associated cracked teeth"), especially where the occlusal loads were intentionally decreased from the implant-supported rehabilitations, thus ensuing increased occlusal load to the adjacent natural teeth.

In this study, a series of 18 cases of implant-associated cracked teeth is presented and analyzed.

\section{Methods}

The following inclusion criteria were applied for case selection:

1. The implants were placed adjacent to natural nonendodontically treated teeth with no pulpal or periradicular pathology.

2. The crack was diagnosed after implant loading, based on a clinical and radiographic evaluation.

3. Occlusal contacts were present between the cracked teeth and the opposing teeth.

4. The crack was confirmed by visual examination using magnification and illumination. The restoration was removed for observation, and the tooth was stained using methylene blue dye when indicated (Kang et al. 2016).

\section{Data collection and analysis}

Data from a series of 18 patients with implant-supported rehabilitation/s who were referred for endodontic treatment between June 2015 and June 2016, with 18 cracked non-endodontically treated teeth that were diagnosed after adjacent implant placement and loading, were retrospectively collected and analyzed.

The following data were recorded for each patient based on the patients' medical records and radiographic examinations: age and sex, the cracked tooth type (categorized to maxillary or mandibular anterior/premolar/molar teeth), the type of coronal restoration, presence of deep periodontal pockets (if the periodontal probing depth was over $4 \mathrm{~mm}$ (Kim et al. 2013)), the pulp diagnosis (American association of endodontics 2013), the number and locations of the dental implants, and the time from implant loading to the diagnosis of the crack.

\section{Results}

Data from a series of 18 cases of cracked teeth that were diagnosed following the placement of adjacent implantsupported rehabilitation were collected and analyzed.

There were 11 (61\%) women and 7 (39\%) men between the ages of 38 and 76 years (average age $=59$ years). Fourteen patients (78\%) were over 50 years.
Nine $(50 \%)$ of the cracked teeth were molar teeth (4 in the maxilla and 5 in the mandible), 7 (39\%) were mandibular premolars, and 2 (11\%) were incisor teeth.

Eleven (61\%) of the cracked teeth had an amalgam restoration. The remaining teeth $(n=7)$ had either a composite restoration $(n=4,22 \%)$, full coverage restoration $(n=2,11 \%)$, or no restoration $(n=1,6 \%)$.

There were nine teeth (50\%) that had deep periodontal pockets at the time of the crack diagnosis. There were no reports in the patient's medical records on deep pockets from the time of implant placement.

There were 14 (78\%) teeth that had vital pulps. The remaining $4(22 \%)$ teeth had pulp necrosis.

In $4(22 \%)$ patients, a single implant was present, in 4 (22\%) patients, two implants were placed, and in 10 (56\%) patient, three or more implants were placed (average $=3$ implants per patient). In 8 (44\%) patients, the implants were located adjacent to the cracked teeth, and in $10(56 \%)$ patients, implants were located at opposing $(n=3,17 \%)$, contralateral $(n=4,22 \%)$, or at multiple sites in the mouth $(n=3,17 \%)$.

In four teeth (22\%), the time from implant loading to the diagnosis of the crack was less than 1 year, in five (28\%) teeth, it was between 1 and 3 years, and in nine (50\%) teeth, it was more than 3 years.

Figure 1 illustrates a case of a cracked 2nd mandibular molar diagnosed following the placement of an implantsupported rehabilitation in the adjacent area.

\section{Discussion}

This study presents and evaluates a series of 18 cases of cracked non-endodontically treated teeth that were diagnosed following the placement of adjacent implantsupported rehabilitation. A recent study (Rosen et al. 2016) reported for the first time on a series of similar cases that occurred in endodontically treated teeth. In addition, in that study, a systematic review of the literature confirmed that this possible complication was never reported in either endodontically or non-endodontically treated teeth (Rosen et al. 2016).

In the present study, strict inclusion and exclusion criteria were applied (Rosen et al. 2016; Tsesis et al. 2010a). At the time of implant placement, the associated teeth had no pulpal or peri-radicular pathology, the presence of opposing teeth and occlusal contacts was confirmed, and the crack was diagnosed after implant loading. In addition, the diagnosis of cracked teeth is not straightforward because the symptoms are diverse, and crack lines may be difficult to identify (Kang et al. 2016). Thus, the detection of the dentinal crack requires a valid gold standard method (Tsesis et al. 2010a; Tsesis et al. 2010b; Mileman and van den Hout 2009). In the present study, the gold standard method selected for verification of the cracked tooth was confirmation by visual examination 

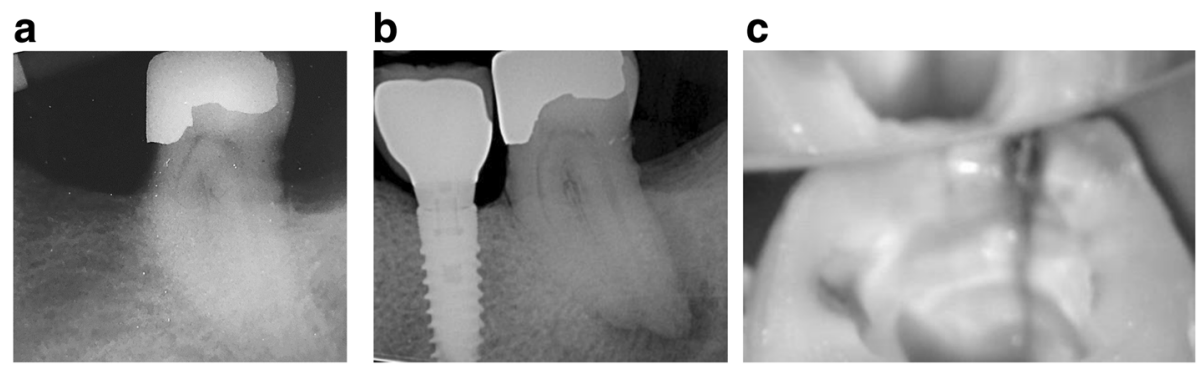

Fig. 1 Cracked 2nd mandibular molar diagnosed following the placement of an implant-supported rehabilitation in the adjacent area. a A mandibular left 2nd molar tooth at time of placement of a mesial implant. There were no clinical or radiographic signs of pathology. b After 14 months, the patient presented with pain and discomfort during mastication in the lower left quadrant. The mandibular left 2nd molar tooth was diagnosed with deep periodontal pockets, pulp necrosis, and apical periodontitis. c Following the removal of coronal restorations, a surgical operating microscope can be used to confirm the presence and extent of the crack

using magnification and illumination and removal of coronal restorations for observation and staining using methylene blue dye when indicated (Kang et al. 2016).

The American Association of Endodontists (AAE) categorizes cracks into five types: craze lines, fractured cusp, cracked tooth, split tooth, and vertical root fracture (VRF) (Kang et al. 2016). Craze lines affect only the enamel, are not associated with any pulpal or periapical pathology, cause no concern beyond esthetics, and thus were not included in the current study. VRFs are rootoriginating fractures that may originate anywhere in the root, occur primarily in endodontically treated teeth (Bakland and Tamse 2015) and were not included in the current study as well. Fractured cusp, cracked tooth, and split tooth describe longitudinal or variations of crownoriginating fractures (Kang et al. 2016; Bakland and Tamse 2015). These were included in the current study and were categorized as one category termed "cracked teeth" (Bakland and Tamse 2015). Thus, although limited in its extent, this case series is the first clinical report of this possible serious complication of implantassociated non-endodontically treated cracked teeth.

In the current study, more cases occurred in female patients (61\%) than in male patients. Cameron (Cameron 1976) reported that the majority (67\%) of cracked teeth were observed in women. However, Kang et al. (Kang et al. 2016), who analyzed the distribution and characteristic features of cracked teeth, reported that cracks were more prevalent among men (61\%) than women. Rosen et al. (Rosen et al. 2016), who specifically evaluated cases of implant-associated VRFs in adjacent endodontically treated teeth, reported on female predominance. Additional studies are required to elucidate the gender effect on the risk of cracked teeth and, specifically, if these cracks are associated with adjacent implant placement.

It had been reported that patients' age may be associated with the risk of cracked teeth, as most cracked teeth are diagnosed in patients aged 50-60 years or even over
60 (Kang et al. 2016; Cameron 1976). The results of the current study support this finding, as most patients in the series $(78 \%)$ were over 50 years old, and the average age was 59 years. Although this effect of age on the risk of cracks is not fully elucidated, it has been suggested that the resistance to fracture of the human dentin may decrease with age due to age-related biomechanical alterations such as loss of dentin elasticity and increased stress fatigue over the years (Kang et al. 2016).

In the current study, most of the teeth (61\%) had an amalgam restoration. However, Kang et al. (Kang et al. 2016) reported that only $6 \%$ of the fractured teeth were restored with amalgam. The difference may be partially explained by the fact that Kang et al. (Kang et al. 2016) included also cases of VRFs that were excluded from the current study. Additional clinical studies are indicated in order to assess the effects of the type of coronal restoration on the development of cracks.

In the current study, nine teeth (50\%) had deep periodontal pockets (more than $4 \mathrm{~mm}$ ). It had been reported that the probing depth may affect the prognosis of cracked teeth (Kim et al. 2013; Kang et al. 2016). Kang et al. (Kang et al. 2016) classified probing depths by $3 \mathrm{~mm}$ intervals and reported that about half of the teeth had probing depths of 3 or more millimeter. They also found that probing depths of more than $6 \mathrm{~mm}$ were a significant clinical factor for the survival of cracked teeth treated with a root canal treatment. Kim et al. (Kim et al. 2013) also reported that the prognosis was less favorable in cracked teeth with a deep probing depth.

In the current study, most teeth had a vital pulp (78\%). Kang et al. (Kang et al. 2016) also reported that the majority of teeth $(58 \%)$ were vital. The remaining 4 (22\%) teeth in the current study had pulp necrosis. When the pulp necrosis is caused by cracks, it may be termed "fracture necrosis" (Berman and Kuttler 2010), a condition that may adversely affect the tooth prognosis.

Due to their PDL, natural teeth have proprioception signaling, which is considered to be a protective mechanism 
(Jackson 2003). However, unlike natural teeth, implants have no PDL; therefore, only muscular and TMJ proprioception mechanisms would signal excessive force (Rosen et al. 2016; Jackson 2003). As a result, the implants' adaptation to occlusal forces is significantly reduced compared to natural teeth (Jackson 2003; Kim et al. 2005). Hence, implants may be more susceptible to occlusal overloading without any patient awareness. Yet, the clinical significance of the occlusal forces to the prognosis of implants is controversial (Rosen et al. 2016; Jackson 2003; Chambrone et al. 2010; Gotfredsen et al. 2001; Misch and Bidez 2005; Heitz-Mayfield et al. 2004).

In order to reduce the risk of implant occlusal overload, a preventive measure has been proposed: the "implant-protective occlusion" (IPO) mechanism. According to this mechanism, the occlusal overload on the implant-supported prosthesis should be decreased in order to increase the chances of successful implant osseointegration (Rosen et al. 2016; Jackson 2003; Misch and Bidez 2005). Since the implant occlusion is part of the entire patient's occlusal system, when the occlusion is designed to minimize the forces applied on the implant, it increases the forces distributed to the adjacent natural teeth (Rosen et al. 2016; Misch 1993; Engelman 1996; Kim et al. 2005; Sekine et al. 1986; Schulte 1995). These forces on adjacent teeth expose these teeth to excessive occlusal loads and thus to risk of developing cracks (Lynch and McConnell 2002; Seo et al. 2012). In the current case series, the majority of patients $(78 \%)$ had multiple implants, potentially significantly increasing the load on the natural dentition (Misch 1993; Engelman 1996; Kim et al. 2005; Sekine et al. 1986; Schulte 1995). It had already been reported that occlusal alterations caused by tooth loss and implant placement may contribute to the development of root fractures in adjacent endodontically treated teeth (Rosen et al. 2016). It is therefore conceivable to assume that such implantrelated occlusal loads may also contribute to the risk of cracks in adjacent non-endodontically treated teeth. However, additional clinical studies are warranted in order to elucidate the exact occlusal interrelations between the implants and the affected cracked teeth.

In this study, $39 \%$ of the cracks were identified in mandibular premolars. Half of the cases were identified in molar teeth $(50 \%)$, of which only $5(28 \%)$ were identified in mandibular molars. This is not consistent with the known classification of matched tooth locations as the more susceptible locations for cracked teeth development. Cameron (Cameron 1976; Cameron 1964) and Kang et al. (Kang et al. 2016) reported that these cracks occur most frequently in mandibular second molars, and that cracked mandibular premolars are rare $(2 \%$ of cases (Kang et al. 2016)). Additional studies are required in order to elucidate if the association to adjacent implants affects the fracture risk distribution, potentially leading to an increased rate of fractures in mandibular premolar teeth.

In the current study, most of the cracked teeth (78\%) were diagnosed more than 1 year after implant loading. The final diagnosis of a cracked tooth may be complicated and delayed since the tooth may be asymptomatic or with non-specific pulpal and periapical symptoms, and since the tooth structure usually appears normal in the radiograph (Cameron 1976; Cameron 1964). In addition, the confirmation of the crack may be complex and requires visual examination by magnification and illumination, removal of coronal restorations for observation, and staining of the crown using appropriate staining materials such as methylene blue dye (Kang et al. 2016).

This study suggests, for the first time, a possible of association between implant-supported rehabilitation and the development of cracks in adjacent nonendodontically treated teeth. However, several independent variables that were not available in this study, such as the type of restoration on the cracked tooth, the time the teeth were initially restored, and the length of time they were in occlusion, need to be controlled in future studies before any conclusion on a direct causal relationship. Furthermore, additional prospective studies with a larger sample are indicated in order to better understand the possible correlation between implant rehabilitation and cracked adjacent teeth, and in order to calculate the incidence of this potential complication.

\section{Conclusions}

This case series is the first clinical report of a possible complication of implant-supported rehabilitation: ensuing cracks of adjacent non-endodontically treated teeth. The most common patient profile found in this series was a woman over 50 years old, having a cracked mandibular premolar tooth, which was diagnosed more than 1 year after reconstruction based on multiple adjacent implants. Additional clinical studies are warranted in order to shed light on this potential complication. Furthermore, a prospective large scale clinical study seems to be recommended.

\section{Funding \\ There was no funding in this study.}

\section{Authors' contributions}

$E R, A T$, IB, and IT designed the study. ER, TG, and SB collected and analyzed the data. ER, TG, CE-N, IB, and IT wrote the paper. IB and IT shared equal contribution. All authors read and approved the final manuscript.

Ethics approval and consent to participate

This study was approved by the Tel Aviv university ethics committee.

Consent for publication

Patient consent was obtained. 


\section{Competing interests}

ER and IT are the Editors-in-chief, and AT and CE-N are advisory board members. The other authors declare no competing interests.

\section{Publisher's Note}

Springer Nature remains neutral with regard to jurisdictional claims in published maps and institutional affiliations.

\section{Author details}

'Department of Endodontology, The Maurice and Gabriela Goldschleger School of Dental Medicine, Tel Aviv University, Tel Aviv, Israel. ²Department of Periodontology and Dental Implantology, The Maurice and Gabriela Goldschleger School of Dental Medicine, Tel Aviv University, Tel Aviv, Israel. ${ }^{3}$ Department of Oral-Rehabilitation, The Maurice and Gabriela Goldschleger School of Dental Medicine, Tel Aviv University, Tel Aviv, Israel.

Received: 16 June 2017 Accepted: 14 August 2017

Published online: 22 August 2017

\section{References}

American association of endodontics - Colleagues for Excellence - Endodontic Diagnosis 2013.

Bakland LK, Tamse A. Categorization of dental fractures. In: Tamse A, Tsesis I, Rosen $\mathrm{E}$, editors. Vertical root fractures in dentistry. 1st ed. Switzerland: Springer International Publishing; 2015. p. 7-28.

Berman LH, Kuttler S. Fracture necrosis: diagnosis, prognosis assessment, and treatment recommendations. J Endod. 2010;36(3):442-6.

Cameron CE. Cracked-tooth syndrome. J Am Dent Assoc. 1964;68:405-11.

Cameron CE. The cracked tooth syndrome: additional findings. J Am Dent Assoc. 1976;93(5):971-5.

Chambrone L, Chambrone LA, Lima LA. Effects of occlusal overload on periimplant tissue health: a systematic review of animal-model studies. J Periodontol. 2010;81(10):1367-78.

Depprich R, Zipprich H, Ommerborn M, Naujoks C, Wiesmann HP, Kiattavorncharoen S, et al. Osseointegration of zirconia implants compared with titanium: an in vivo study. Head Face Med. 2008;4:30.

Engelman MJ. Occlusion. Clinical decision making and treatment planning in osseointegration. 1st ed. Chicago: Quintessence Publishing Co; 1996. p. 169-76.

Goodacre CJ, Spolnik KJ. The prosthodontic management of endodontically treated teeth: a literature review. Part I. Success and failure data, treatment concepts. J Prosthodont. 1994;3(4):243-50.

Gotfredsen K, Berglundh T, Lindhe J. Bone reactions adjacent to titanium implants subjected to static load of different duration. A study in the dog (III). Clin Oral Implants Res. 2001;12(6):552-8.

Heitz-Mayfield L, Schmid B, Weigel C, Gerber S, Bosshardt DD, Jonsson J, et al. Does excessive occlusal load affect osseointegration? An experimental study in the dog. Clin Oral Implants Res. 2004;15(3):259-68.

Jackson BJ. Occlusal principles and clinical applications for endosseous implants. J Oral Implantol. 2003;29(5):230-4.

Kang SH, Kim BS, Kim Y. Cracked teeth: distribution, characteristics, and survival after root canal treatment. J Endod. 2016;42(4):557-62.

Kim SY, Kim SH, Cho SB, Lee GO, Yang SE. Different treatment protocols for different pulpal and periapical diagnoses of 72 cracked teeth. J Endod. 2013; 39(4):449-52.

Kim Y, Oh TJ, Misch CE, Wang HL. Occlusal considerations in implant therapy: clinical guidelines with biomechanical rationale. Clin Oral Implants Res. 2005;16(1):26-35.

Koyano K, Esaki D. Occlusion on oral implants: current clinical guidelines. J Oral Rehabil. 2014;

Lynch CD, McConnell RJ. The cracked tooth syndrome. J Can Dent Assoc. 2002;68(8):470-5.

Mericske-Stern R, Grutter L, Rosch R, Mericske E. Clinical evaluation and prosthetic complications of single tooth replacements by non-submerged implants. Clin Oral Implants Res. 2001;12(4):309-18.

Mileman PA, van den Hout WB. Evidence-based diagnosis and clinical decision making. Dentomaxillofac Radiol. 2009;38(1):1-10.

Misch CE. Occlusal considerations or implant supported prostheses. In: Misch CE, editor. Contemporary implant dentistry. 1st ed. St. Louis: Mosby; 1993. p. 705-33.

Misch CE, Bidez MW. Occlusal considerations for implant-supported prosthesis: implant protective occlusion. Dental implant prosthetics. St.Louis: Elsevier/ Mosby; 2005. p. 472-507.
Morgano SM, Brackett SE. Foundation restorations in fixed prosthodontics: current knowledge and future needs. J Prosthet Dent. 1999;82(6):643-57.

Novaes AB Jr, de Souza SL, de Barros RR, Pereira KK, lezzi G, Piattelli A. Influence of implant surfaces on osseointegration. Braz Dent J. 2010;21(6):471-81.

Robbins JW. Guidelines for the restoration of endodontically treated teeth. J Am Dent Assoc. 1990;120(5):558-60. 62 passim

Rosen E, Beitlitum I, Tamse A, Taschieri S, Tsesis I. Implant-associated vertical root fracture in adjacent endodontically treated teeth: a case series and a systematic review. J Endod. 2016;42(6):948-52.

Schulte W. Implants and the periodontium. Int Dent J. 1995;45(1):16-26.

Sekine $\mathrm{H}$, Komiyama Y, Potta H, Yoshida K. Mobility characteristics and tactile sensitivity of osseointegrated fixture-supporting systems. In: van-Steenberghe D, Albrektsson T, Branemark PI, Henry PJ, Holt R, Liden G, editors. Tissue integration in oral and maxillofacial reconstruction. Amsterdam: Excerpta Medica; 1986. p. 326-32.

Seo DG, Yi YA, Shin SJ, Park JW. Analysis of factors associated with cracked teeth. J Endod. 2012;38(3):288-92.

Tsesis I, Rosen E, Tamse A, Taschieri S, Kfir A. Diagnosis of vertical root fractures in endodontically treated teeth based on clinical and radiographic indices: a systematic review. J Endod. 2010a;36(9):1455-8.

Tsesis I, Nemkowsky CE, Tamse E, Rosen E. Preserving the natural tooth versus extraction and implant placement: making a rational clinical decision. Refuat Hapeh Vehashinayim. 2010b;27(1):37-46. 75

\section{Submit your manuscript to a SpringerOpen ${ }^{\circ}$ journal and benefit from:}

- Convenient online submission

- Rigorous peer review

- Open access: articles freely available online

- High visibility within the field

- Retaining the copyright to your article

Submit your next manuscript at $>$ springeropen.com 